Original Research Paper

\title{
Asymptomatic COVID-19 Patients: Experience and Lessons from a Developing Country
}

\author{
${ }^{1}$ Sylvie Kolani, ${ }^{1}$ Houda Alami, ${ }^{3}$ El Harch ibtissam, ${ }^{2}$ Said Boujraf, ${ }^{1,2}$ Meryem Haloua, \\ ${ }_{1,2}$ Youssef Alaoui Lamrani, ${ }^{3}$ Samira El Fakir, ${ }^{4}$ Mounia Serraj, ${ }^{4}$ Bouchra Aamara, \\ ${ }^{1,2}$ Meryem Boubbou, ${ }^{1,2}$ Mustapha Maaroufi and ${ }^{1,2}$ Badreeddine Alami
}

\author{
${ }^{1}$ Department of Radiology, Hassan II University Hospital, Fez, Morocco \\ ${ }^{2}$ Laboratory of Neurosciences, Faculty of Medecine and Pharmacy of Fez, \\ Sidi Mohamed ben Abdellah University, Fez, Morocco \\ ${ }^{3}$ Department of Epidemiology, Hassan II University Hospital, Fez, Morocco \\ ${ }^{4}$ Department of Pneumology, Hassan II University Hospital, Fez, Morocco
}

Article history

Received: 11-10-2020

Revised: 18-01-2021

Accepted: 03-02-2021

Corresponding Author:

Sylvie Kolani

Department of Radiology,

Hassan II University Hospital,

Fez, Morocco

Email: kolanisylvie@gmail.com

\begin{abstract}
The world is facing a pandemic due to the SARS-Coronavirus 2 , since late 2019. Many questions remain unanswered regarding the disease course. Key amongst its talking point is the case of asymptomatic patients. This potentially jeopardizes infection control strategy as asymptomatic cases are difficult to identify and hence difficult to isolate. Our study intends to define the clinical and radiological features of asymptomatic COVID-19 cases, the disease course as well as highlight the role of chest CT in its management. This is a monocentric study involving 114 asymptomatic adults admitted in our COVID-19 Unit. Clinical, radiological and laboratory findings were retrospectively analyzed. Chi-squared, Fisher exact test and the student test were used for statistical analysis. Asymptomatic patients represent $51.81 \%$ of patients. There was a slight male predominance with a mean age of 37.64 years. Patients with abnormal CT had a longer hospital stay than those with unremarkable CT and even more so were older. None of the patients presented severe or critical extension of parenchymal lesions. Only two patients $(4.54 \%)$ with normal CT on admission presented with abnormalities on control CT. Cases with worsening CT were older with bilateral pulmonary involvement. All patients remained asymptomatic on treatment. Even when asymptomatic, COVID-19 patients present mild lung lesions. The positivity of the initial chest CT imaging is directly correlated to the disease course. Older patients with bilateral pulmonary lesions are more likely to worsen and should be closely monitored. Moreover, it is safe to manage asymptomatic patients with normal $\mathrm{CT}$ in a non-hospital setting.
\end{abstract}

Keywords: COVID-19-Asymptomatic Patients-Clinical CharacteristicsCT-Prognosis

\section{Introduction}

Since December 2019, the COVID-19 infection caused by a SARS-coronavirus 2 has become a major public health concern, rapidly growing into a pandemic. Health professionals and scientists have been working to find the most effective treatment guidelines as well as ways to prevent the spread of the disease. It is widely believed that a first wave of the pandemic has grinded to a halt and the world is facing a second wave of infections. Africa on the other hand, has been relatively spared by the first wave yet it is not immune to a second one. One of the seed of this second wave is undoubtedly, asymptomatic COVID-19 patients. This category of patients jeopardizes infection control strategy as they are difficult to identify, hence difficult to isolate and manage. Few authors have 
looked into this category of patients with still many grey areas in literature. This study seeks to identify clinical and radiological features of asymptomatic patients as well as factors that influence disease course while highlighting the role of chest $\mathrm{CT}$ in the management of these patients.

\section{Materials and Methods}

\section{Patients}

We conducted a monocentric retrospective study involving 114 asymptomatic adults with Reverse Transcriptase-Polymerase Chain Reaction (RT-PCR) confirmed SARS-coronavirus 2 infection. Local Institutional Review Board approval and written informed consent was obtained from all patients prior to the study. Our center was at the time the only COVID-19 unit of the city, for PCR test as well as management of patients. The national management protocol includes the hospitalization of all COVID-19 patients. All asymptomatic patients admitted during the time of the study were included. 106 symptomatic cases presenting with either fever, respiratory symptoms, anosmia or flu-like syndrome (asthenia, myalgia), occurring in up to 15 days before admission, were excluded from the study. Children under 16 years were also excluded. All patients were admitted in the COVID19 Unit until two consecutive negative RT-PCR tests with clinical assessment daily. Patients were treated according to the national treatment protocol including an association of Azithromycin for 05 days and Chloroquine for 10 days as first line or Lopinavir/Ritonavir for 10 days as second line treatment.

Clinical characteristics such as the gender, the age, the comorbidities, course of disease were recorded during admission. Additionally, epidemiologic characteristics regarding the context of infection, laboratory findings notably white blood cell count and C-reactive protein level were also analyzed. Finally, chest CT results were reviewed.

\section{Images Acquisitions and Analysis}

A chest CT without contrast was performed in all patients on admission. The first control was performed on the tenth day after admission. The acquisition covered the diaphragmatic cupola to the superior thoracic aperture with patient supine holding his breath in end inspiration state. The parameters used for the scanning protocol were as follow: Tube voltage $120 \mathrm{kV}$, section thickness after reconstruction of $2.5 \mathrm{~mm}$ reconstructed in 1.25 $\mathrm{mm}$. The reconstructed CT images were transmitted to the workstation and Picture Archiving and Communication Systems (PACS) for multiplanar reconstruction post-processing.
The images were viewed in lung and mediastinal window by two seniors radiologists who are consultants specialized in thoracic imaging. Parenchymal lesions were classified according to the following pattern: Patchy or nodular ground glass opacities with or without intralobular reticular lines, consolidations with or without halo or reverse halo sign, sub-pleural lines. Also, lesions were categorized according to their bilaterality and extension; and classified as follows: Minor extension when the lesion involved less than $10 \%$ of the lung parenchyma, moderate extension when between 11 to $25 \%$, extensive lesions between 26 to $50 \%$, as severe extension when between 51 to $75 \%$ and critical extension when more than $75 \%$ of lung parenchyma was affected. Data were analyzed using the Statistical Package for the Social Sciences (SPSS) version 25. Categorical variables were reported as numbers and percentages. Continuous variables were reported as means accompanied by their standard deviation. The chi-squared or Fisher exact test were used for categorical variables whereas the student test was used to compare means. A p value $<0.05$ was considered as statistically significant.

\section{Results}

\section{Clinical Features of Patients}

Out of 220 patients admitted during the period of our study, $51.81 \%(\mathrm{n}=114)$ were asymptomatic. There were three groups of patients according to the circumstances of contamination:

- Groupe 1 includes patients in professional clusters which represent $21.05 \%(n=24)$ of all patients

- Groupe 2 involves patients in familial clusters representing $29.82 \%(n=34)$

- Groupe 3 constitutes contacts of confirmed COVID-19 patients representing $49.12 \%(n=56)$ of the population

The mean age of the patients was 37.6 years with a range of 16 to 87 years. $46.5 \%(n=53)$ of patients were female and $53.5 \%(\mathrm{n}=61)$ were male with a male/female ration of 1.2.

All our patients were hospitalized until two negative PCR tests at least $24 \mathrm{~h}$ apart. The average hospital stay was 21.85 days. On admission, none of our patients presented with respiratory symptoms or any common COVID-19 related symptoms. 11 patients (9.64\%) presented side effect of treatment as nausea, vomiting, diarrhea and tremors. One of the patients presented with cardiac side effects due to Chloroquine which necessitated its subsequent removal. All the patients had a favorable outcome with no deaths recorded. 


\section{Imaging and Laboratory Findings}

Initial chest $\mathrm{CT}$ scan found abnormalities in 48 patients which represent $42.10 \%$. The $66(57.89 \%)$ others patients presented with normal initial chest CT scan.

The most common pattern on CT was Ground-Glass Opacities (GGO) present in 41 patients (85.41\%), including simple GGO in 23 cases $(47.9 \%)$. GGO was associated with fine reticulations determining a «Crazy paving » pattern in 05 cases $(10.41 \%)$, with consolidations in 12 cases $(29.26 \%)$. The second frequent CT feature was consolidations, present in 18 cases (37.5\%). Others features found were subpleural lines and a spontaneous pneumomediastinum in a single patient [Table 1].

Heterogeneous distribution of the lung lesions was observed. However, the most common location was peripheral and subpleural which was observed in 41 patients (85.41\%). In 07 patients $(14.58 \%)$ the localization of abnormalities was both peripheral and central. The lesions were bilateral in 31 patients $(64.58 \%)$.

Regarding the extent of pulmonary lesions, 24 (50\%) patients had minimal lung involvement lesser than $10 \%$, while $17(35.41 \%)$ patients showed moderate lung involvement ranging between 11 to $25 \%, 07$ (14.58\%) had an extensive lung involvement ranging from 26 to $50 \%$. None of the patient presented severe or critical extension (greater than 50\% lung involvement).

Patients with extensive pulmonary lesion were hospitalized longer than those with minimal lesions: 22.66, 24.85 and 25.41 days for minimal, moderate and extensive involvement respectively.

90 patients had a follow-up CT: 46 out of 48 patients that had pathological initial CT and, 44 of the 66 patients that presented with normal initial CT. 02 patients with an initial normal CT presented with moderate pulmonary lesion extension on day 10 of admission. All the other patients with normal initial CT did not show any lesions on follow-up CT. Regarding the second group of patients with initial pathological CT: Partial resorption was observed in 28 (60.08\%), total regression of lesions in $n=08(17.39 \%)$, stability in $n=05(10.86 \%)$ and worsening in 05 patients $(10.86 \%)$.

Laboratory results of patients who exhibited progression of lesions on the control CT were unremarkable: None of them had lymphopenia or positive C-reactive protein [Table 2].

\section{Comparison of Patients with Normal CT and Pathological CT Table 3}

The mean age of patients with a normal CT was 36.10 years and for those with pathological CT was 39.75 years, although the difference was not statistically significative. Also, there was no particularity considering the gender. On the other hand, patients with abnormal CT were hospitalized longer than patients with normal CT scan on admission $(p=0.036)$. Moreover, patients with abnormal CT were more likely to present new pulmonary lesions.

\section{Particularities of "Progressors" Group Patients on CT Table 4}

The mean age of the group was 45.42 years, which is 7.78 years older than the mean age of the patients of our series, 9.3 years older than patient with initial normal chest $\mathrm{CT}$ and 6.25 years older than patients that present stability/regression of lesions on the first control. Also, they had more bilateral pulmonary lesion on the initial CT compared to all patients ( $80 \mathrm{Vs} 64.58 \%$ ) and compared to patients who present stable or regression of initial parenchymal lesions (80 Vs $63.41 \%$ ).

Table 1: Comparison of clinico-radiological features of our series with series in the litterature

\begin{tabular}{llllll}
\hline & & Our Series & Tabata et al. $(2020)$ & Meng et al. (2020) & Hu et al. (2020) \\
\hline Population & & $114 / 220$ & $43 / 104$ & 58 & 24 \\
Gender & Women & $(51.8 \%)$ & $(41 \%)$ & & \\
& Men & $53(46,5 \%)$ & $18(55 \%)$ & $32(55.2 \%)$ & $16(66.6 \%)$ \\
Age (years) & Mean & $37(53,5 \%)$ & $15(45 \%)$ & $26(44.8 \%)$ & $8(33.33 \%)$ \\
& Range & $16-87 \pm 14,8$ & 70 & $42 \pm 16.56$ & 32.5 \\
Remained COVID-19 & & $114(100 \%)$ & $33(32 \%)$ & $42(72 \%)$ & $5-95$ \\
symptoms free & & & & $19(79.16 \%)$ \\
Positive CT & & $48(42.10 \%)$ & $17(52 \%)$ & $100 \%$ & $17(70.8 \%)$ \\
Lesions on CT & GGO & $41(85.41 \%)$ & $11(33 \%)$ & $55(94,8 \%)$ & $12(50 \%)$ \\
& Consolidations & $18(37.5 \%)$ & $6(18 \%)$ & $3(5.2 \%)$ & 0 \\
& Crazy paving & $5(10.41 \%)$ & $2(6 \%)$ & $7(12 \%)$ & 0 \\
& Nodular lesions & 0 & - & 0 \\
Bilateral pulmonary lesions & $2(6 \%)$ & $6(10.3 \%)$ & 0 \\
Hospital stay (days) & Sub-pleural lines & $6(12.5 \%)$ & - & - & $5(20.8 \%)$ \\
Progression on CT control & Stipes shadows & 0 & - & $24(41,4 \%)$ & - \\
\hline
\end{tabular}


Table 2: Comparison of clinical features and disease course between patients with normal CT Vs abnormal CT

\begin{tabular}{|c|c|c|c|c|}
\hline & & Normal CT findings & Abnormal CT findings & Pvalue \\
\hline Number & & $66 / 114$ & $48 / 114$ & \\
\hline Mean age (years) & & $36,11 \pm 15,33$ & $39,75 \pm 13,93$ & 0,196 \\
\hline Male & & $39(59,09 \%)$ & $22(45,83 \%)$ & 0,186 \\
\hline Female & & $27(40,9 \%)$ & $26(54,17 \%)$ & \\
\hline Hospital stay (days) & & $20,32 \pm 8,473$ & $23,96 \pm 9,733$ & 0,036 \\
\hline \multirow[t]{3}{*}{ Evolution on 10th day } & Regression & - & $36(78,26 \%)$ & 0,000 \\
\hline & Stability & $42(95,45 \%)$ & $5(10,86 \%)$ & \\
\hline & Progression & $2(4,54 \%)$ & $5(10,86 \%)$ & \\
\hline
\end{tabular}

Table 3: Clinico-radiological features and laboratory findings of worsening patients on the seventh day of admission CT control

\begin{tabular}{|c|c|c|c|c|c|c|c|}
\hline Gender & Age (years) & Comorbidities & C-reactive proteine & Lymphocyte & $\begin{array}{l}\text { Pulmonary lesions } \\
\text { on initial CT }\end{array}$ & $\begin{array}{l}\text { Initial } \\
\text { extension }\end{array}$ & $\begin{array}{l}\text { Extension on } \\
\text { day } 7 \mathrm{CT}\end{array}$ \\
\hline$\overline{\text { Male }}$ & 55 & None & Normal & Normal & Normal & - & Moderate \\
\hline Male & 55 & None & Normal & Normal & Normal & - & Moderate \\
\hline Male & 31 & None & Normal & Normal & Bilateral consolidation $+\mathrm{GGO}$ & Minor & Moderate \\
\hline Female & 54 & None & Normal & Normal & Bilateral nodular GGO & Moderate & Extensive \\
\hline Male & 48 & None & Normal & Normal & Bilateral patchy GGO & Extensive & Extensive \\
\hline Female & 48 & None & Normal & Normal & Bilateral GGO+ consolidation & Moderate & Extensive \\
\hline
\end{tabular}

Table 4: Comparison of clinico-radiological features and disease course between worsening patients on the first CT control vs patient with abnormal initial CT

\begin{tabular}{llll}
\hline & & Worsening patient with abnormal initial CT & Patients with abnormal initial CT \\
\hline Male & & $40 \%$ & $45,83 \%$ \\
Female & $60 \%$ & $54,17 \%$ \\
Mean age (years) & & 45,42 & 39,75 \\
Extension of lesion & Minor & $40 \%$ & $50 \%$ \\
& Moderate & $40 \%$ & $35,41 \%$ \\
& Extensive & $20 \%$ & $14,58 \%$ \\
Type of lesions & GGO & $100 \%$ & $85,41 \%$ \\
& Consolidation & $40 \%$ & $37,5 \%$ \\
Bilaterality of lesions & & $80 \%$ & $64,58 \%$ \\
Hospital stay (days) & & 27,4 & 23,96 \\
\hline
\end{tabular}

\section{Discussion}

COVID-19 has been demonstrated to spread from person to another, primarily through respiratory droplets. The most common clinical manifestations are fever, cough, myalgia or fatigue, anosmia and ageusia. Less common symptoms include gastrointestinal symptoms such as diarrhea, nausea and vomiting. Other atypical symptoms such as headache and hemoptysis have been previously report (Zu et al., 2020).

A certain group of patients do not present any symptoms and represent the asymptomatic positive COVID-19 patients. This category of patient are seeds to clusters and thus hinder infection control and prevention strategies. They could potentially jeopardize patients tracking, protection of health professionals and the efficiency on popular COVID-19 and non-COVID 19 pathways used in hospitals setting. The percentage of asymptomatic COVID-19 patients is not well understood; it varies between 5 and $80 \%$ (CEBM, 2020). In our series, they represent half of the admissions during the study period with a slight male predominance: 1.2: 1 .
Even when asymptomatic, COVID-19 patients could present pulmonary abnormalities on $\mathrm{CT}$ which was the case in 4/10 patients in our series, with up to one-third presenting with consolidations. This corresponds, according to the CT imaging timeline of the infection, to at least the second phase (5-8 days) of the disease (Pan et al., 2020). None of the patients presented with severe or critical extension of pulmonary lesions on admission or during their hospitalization suggesting that alveolar-interstitial lesions are moderate-to-limited in asymptomatic patients.

Comparing patients with normal CT to those with abnormal CT, the former was slightly younger with no gender predominance. However, patients with abnormal CT were hospitalized longer than patients with normal CT scan on admission $(p=0.036)$. Moreover, patients with abnormal CT were more likely to present new pulmonary lesions. Generally, outcome of asymptomatic COVID-19 is favorable as all the patients remained asymptomatic with no deaths recorded. More than threequarters of the patients had partial or total resorption of lesions on follow up CT 10 days after admission. A few (07 out of 90) patients progressed on the follow up CT and 
were mostly (5/7) patients with initial abnormal CT. Further analysis of the «progressors » group highlighted a relatively older population without any particularity in terms of gender, comorbidities, or laboratory findings. The additional particularity observed is that they exhibited more bilateral pulmonary lesions compared to all patients and compared to patients who present with stable or regression of the initial parenchymal lesions.

Some interesting findings could be evidenced in literature [Table 1].

Regarding the sex ratio, data from other series showed the contrary, a female predominance with a sex ratio varying between 0.5 and 0.8 (Tabata et al., 2020; Meng et al., 2020, Hu et al., 2020),

The positivity of the initial CT in asymptomatic patients in others series is higher than in our study and varies from 52 to $100 \%$ [Table 1]. This could be explained by the fact that our population is larger than the other studies in literature we compare our results to. However, these results highlight a major underlying diagnostic issue. The average sensibility of the chest CT in the diagnosis of asymptomatic patients indicate that even a combination of negative $\mathrm{CT} /$ clinical setting cannot rule out the disease. Owing to the fact that the reliability of viral RNA swabs in clinical practice depends on the site and quality of sampling, the stage of disease and degree of viral multiplication or clearance (Nandini et al., 2020; Wölfel et al., 2020; Wang et al., 2020), there is a significant proportion of patients who will not be diagnosed using a combination of clinical setting/CT/PCR. Indeed, one-third of our patients $(65 / 220)$ had negative CT and remained asymptomatic until discharge. On the other hand, (Ling et al., 2020) reported that only 4 out of 34 patients remained asymptomatic during the disease course with normal CT up to 14 days after admission. It is important to note that this study included both asymptomatic and symptomatic patients.

Among other factors, age has been identified as a risk factor for a more severe disease course and mortality. In this vein, the age difference between patients with normal CT versus abnormal $\mathrm{CT}$ is an important observation as it suggests that older patients have more pulmonary damage. Kronbichler et al. (2020); Hu et al., (2020) demonstrated that the age difference between the two groups is statistically significant with a $p$ value respectively of 0.013 and 0.012 . These two studies included children whereas children were excluded in our population.

Another interesting point that needs to be emphasized is the characteristics of the «progressors» group. The older age of patients observed in our study is consistent with observations made on the Diamond princess cruise ship. Indeed, the authors found that comparing patients with mild disease to patients with severe COVID-19 infection, the latter were older ( $73 \mathrm{Vs} 60$ years, $p=0.028)$. Additionally, they had more frequent consolidation on chest CT (46 Vs $21 \%, p=0.035)$, lymphopenia (57 Vs $23 \%, p=0.0055)$ on admission (Tabata et al., 2020). However, there is limitation in interpretation, due the fact that even though, the patients in our series progressed on CT, they remained asymptomatic. This could be attributed to the efficacy of the treatment protocol that our patients followed.

Considering the fact that any patient declared positive was hospitalized during the period of our study and given that the patients in the series were recruited solely based on their asymptomatic character, the population of the series represented accurately the general population allowing us to extrapolate our results.

Since all the patients were put on treatment, their outcome cannot be exclusively attributed to their asymptomatic character; and on the other hand the role of the treatment is yet to be demontrated.

Overall, our study raises questions that merit further research notably the role of $\mathrm{CT}$ as a screening tool for COVID-19 patients and the benefits of treating asymptomatic patients.

\section{Conclusion}

The presence in asymptomatic COVID-19 patients of mild pulmonary lesions, the patient's age as a primary risk factor for severity and progression of pulmonary lesions, as well as the direct correlation between the positivity of initial CT scan and the duration of evolution add to a growing body of evidence on this category of patients. Also, this study illustrates the pivotal role of chest CT in the management of asymptomatic COVID-19 patients. Based on our evidence it is recommended to manage asymptomatic patients with normal CT in non-hospital settings, though elderly patients with bilateral lesions on initial CT should be closely monitored.

\section{Acknowledgement}

Our sincere gratitude goes to Dr Saeed Abdulrazak for proof reading this article.

\section{Funding Statement}

This manuscript was prepared independently without any funding support.

\section{Authors Contributions}

Sylvie Kolani and Badreeddine Alami: Study design, data analysis, writing.

Houda Alami and Sylvie Kolani: Data collection.

Samira El Fakir and El Harch Ibtissam: Data analysis.

Said Boujraf, Meryem Haloua, Youssef Alaoui Lamrani, Mounia Serraj, Bouchra Aamara, Meryem Boubbou and Mustapha Maaroufi: Review. 


\section{Ethics}

The authors declare no ethical issue. Institutional Review Board approval and informed consent were obtained from patients.

\section{References}

CEBM. (2020), COVID-19: What proportion are asymptomatic? University of Oxford, Centre for Evidence-Based Medicine. https:/www.cebm.net/COVID-19/COVID-19-whatproportion-are-asymptomatic/

Hu, Z., Song, C., Xu, C., Jin, G., Chen, Y., Xu, X.,... \& Shen, H. (2020). Clinical characteristics of 24 asymptomatic infections with COVID-19 screened among close contacts in Nanjing, China. Science China Life Sciences, 63(5), 706-711. https://link.springer.com/article/10.1007/s11427020-1661-4

Kronbichler, A., Kresse, D., Yoon, S., Lee, K. H., Effenberger, M., \& Shin, J. I. (2020). Asymptomatic patients as a source of COVID-19 infections: A systematic review and meta-analysis. International Journal of Infectious Diseases, 98, 180-186. https://doi.org/10.1016/j.ijid.2020.06.052

Ling, Z., Xu, X., Gan, Q., Zhang, L., Luo, L., Tang, X., \& Liu, J. (2020). Asymptomatic SARS-CoV-2 infected patients with persistent negative CT findings. European Journal of Radiology, 126. https://www.ejradiology.com/article/S0720048X(20)30145-5/abstract

Meng, H., Xiong, R., He, R., Lin, W., Hao, B., Zhang, L.,... \& Geng, Q. (2020). CT imaging and clinical course of asymptomatic cases with COVID-19 pneumonia at admission in Wuhan, China. Journal of Infection, $\quad 81(1), \quad$ e33-e39. https://doi.org/10.1016/j.jinf.2020.04.004

Nandini, S., Sundararaj, S. J., \& Akihide, R. (2020). Interpreting Diagnostic Tests for SARS-CoV-2 AuJAMA.

https://jamanetwork.com/journals/jama/articleabstract/2765837
Pan, F., Ye, T., Sun, P., Gui, S., Liang, B., Li, L.,... \& Zheng, C. (2020). Time course of lung changes on chest CT during recovery from 2019 novel coronavirus (COVID-19) pneumonia. Radiology. https://www.ncbi.nlm.nih.gov/pmc/articles/PMC723 $3367 /$

Tabata, S., Imai, K., Kawano, S., Ikeda, M., Kodama, T., Miyoshi, K.,.. \& Tamura, K. (2020). Clinical characteristics of COVID-19 in 104 people with SARS-CoV-2 infection on the Diamond Princess cruise ship: a retrospective analysis. The Lancet Infectious Diseases, 20(9), 1043-1050. https://doi.org/10.1016/S1473-3099(20)30482-5

Wang, W., Xu, Y., Gao, R., Lu, R., Han, K., Wu, G., \& Tan, W. (2020). Detection of SARS-CoV-2 in different types of clinical specimens. JAMA, 323(18), 1843-1844. https://jamanetwork.com/journals/jama/articleabstract/2762997

Wölfel, R., Corman, V. M., Guggemos, W., Seilmaier, M., Zange, S., Müller, M. A.,... \& Wendtner, C. (2020). Evaluación virológica de pacientes hospitalizados con COVID-2019. Naturaleza. Publicado en línea en abril, 1. http://colbiosa.com.ar/wpcontent/uploads/2020/05/Documento-26.pdf

Zu, Z. Y., Jiang, M. D., Xu, P. P., Chen, W., Ni, Q. Q., Lu, G. M., \& Zhang, L. J. (2020). Coronavirus disease 2019 (COVID-19): A perspective from China. Radiology, 296(2), E15-E25. https://pubs.rsna.org/doi/abs/10.1148/radiol.202020 0490
Abbreviations
COVID-19: Coronavirus Disease 2019
CT: Comptuted Tomography
GCO: $\quad$ Ground glass Opacity
SARS CoV 2: Severe Acute Respiratory Syndrome Coronavirus 2
RT-PCR: Reverse Transcriptase-Polymerase Chain Reaction 